\title{
Further Investigations on Doubly Excited Four-Electron lons: Beam-Foil Experiment and MCDF Theory
}

\author{
J. H. Blanke, B. Fricke and D. Heinemann \\ Fachbereich Physik, Universität Kassel, D-3500 Kassel, Germany \\ and \\ P. H. Heckmann, G. Möller and E. Träbert \\ Experimentalphysik III, Ruhr-Universität Bochum, Postfach 1021 48, D-4630 Bochum 1, Germany
}

Received April 12, 1991; accepted June 26, 1991

\begin{abstract}
In continuation of our previous work on the quintet transitions $1 s 2 s 2 p^{2}{ }^{5} P_{-}$ I $s 2 s 2 p 3 d^{5} p^{o},{ }^{5} D^{0}$, results on other $n=2-n^{\prime}=3$ quintet transitions for elements $\mathrm{N}, \mathrm{O}$ and $\mathrm{F}$ are presented. Assignments have been established by comparison with Multi-Configuration Dirac-Fock calculations. High spectral resolution on beam-foil spectroscopy was essential for the identification of most of the lines. For some of the quintet lines decay curves were measured, and the lifetimes extracted were found to be in reasonable agreement with MCDF calculations.
\end{abstract}

\section{Introduction}

Doubly-excited states with four electrons are known to be populated in the beam-foil light source [1]. The optical transition $1 s 2 s 2 p^{2}{ }^{5} P-1 s 2 p^{3}{ }^{5} S^{\circ}$ which connects the two lowest quintet states has been correctly identified in the spectra of a number of ions ( $\mathrm{Li}^{-}$[2], Be I [3], B Ii [4], C III, N IV, O V [5], F VI [6] and Ne VII [7], tentatively also for Mg IX [8]). Theoretical work, with results in good agreement with experiment, has been reported on this transition by various authors [9-11]. In addition, the transitions Be I $1 s 2 s 2 p 3 p{ }^{5} P$ $1 s 2 p^{2} 3 p^{5} S^{\circ}$ and $1 s 2 s 2 p 4 p{ }^{5} P-1 s 2 p^{2} 4 p{ }^{5} S^{\circ}$ have been observed $[12,13]$. In an earlier paper [14] we reported the identification of the $1 s 2 s 2 p^{2}{ }^{5} P-1 s 2 s 2 p 3 d d^{5} P^{\circ},{ }^{5} D^{\circ}$ transitions, for elements $\mathrm{N}, \mathrm{O}, \mathrm{F}$ and $\mathrm{Mg}$, which have been identified by comparing experimental transition energies with theoretical ones obtained with the Multi-Configuration Dirac-Fock program of Desclaux $[15,16]$.

Here we concentrate on other $n=2-n^{\prime}=3$ quintet transitions for the elements $\mathrm{N}, \mathrm{O}$ and $\mathrm{F}$. As an example, an energy level scheme of the quintet states and transitions in question is given in Fig. 1, for the case of oxygen. One should mention that the doubly excited quintet states lie in the continuum above the ionization limit $1 s^{2} 2 s$ of singly excited four-electron states. They are stable against E1-dipole transitions to single excited states because their maximal spin quantum number $S=2$ canot be obtained with the $1 s^{2}$-core. The same argument applies to the autoionization to $1 s^{2} 2 s \varepsilon l{ }^{1.3} L$ continuum states. Further, all quintet states considered lie below the $1.52 s 2{ }^{4} P^{\circ}$ limit, thus they are also stable against Coulomb autoionization to $1 s 2 s 2 p \varepsilon l{ }^{5} L$ continuum states. Therefore optical transitions between these quintet states might be observable. Besides spectra for wavelength determination, decay curves were recorded at the spectral positions of quintet candidate lines as far as measurements were not hindered by strong blends.
In the theoretical part below we show that for the quintet states with a $1 s 2 s 2 p$ core a treatment including the mixing of all states of a given complex is sufficient. This is in contrast to those $n=3$-states with a $1 s 2 p^{2}$ core for which mixing with $1 s 2 s 2 p 4 l{ }^{5} L$ states has to be considered, at least for the elements $\mathrm{N}, \mathrm{O}$ and $\mathrm{F}$. Experimental evidence for this can be found in the wavelength and lifetime data.

After a brief discussion of the theoretical method and the experimental facilities we discuss line identifications and lifetime measurements for the $n=2-n^{\prime}=3$ quintet transition.

\section{Theory}

For our calculations the latest version of the MultiConfiguration Dirac-Fock program [16] was used. There are three stages of theoretical descriptions.

The first step is to include into the basis of $j j$-coupled states only those that originate from a single non-relativistic configuration. The coupling refers to "good" quantum number $J$ of the total angular momentum. Misleadingly we call this the "Single-configuration" or "SC"-approach. An isoelectronic comparison between the results of this simple approach and experimenal results already is a powerful tool for identifying transitions in spectra [17].

At the next stage, all configurations of a complex are included in the basis. This approach permits, for example, to describe the mixing between $1 s 2 s 2 p 3 d^{5} D^{\circ}$ and $1 s 2 p^{2} 3 p{ }^{5} D^{\circ}$. For light elements (and thus $L S$-coupling) the mixing with the configuration $1 s 2 s 2 p 3 s$ is not important, because no ${ }^{5} D^{\circ}$ state can be formed in the latter one. Such calculations in the complex include the linear part of the correlation energy [18]. Further constant contributions can be explained by including configurations from outside the complex. At present we do not attempt to calculate these further contributions; instead the difference between experimental and theoretical transition energies is evaluated along the isoelectronic sequence and its regularity is used to check on possible candidate lines.

This approach is useful and valid as far as no so-called "near perturbers" with slight changed core-configurations from other Rydberg series occur. In the case of the quintet transitions, this is in fact a problem for $1 s 2 p^{2} 3 l$ configurations. They mix strongly with configurations of the form $1 s 2 s 2 p 4 l$ for the elements $\mathrm{N}, \mathrm{O}$ and $\mathrm{F}$, as discussed in this work. The influence of the mixing might be minor for $F$ and 


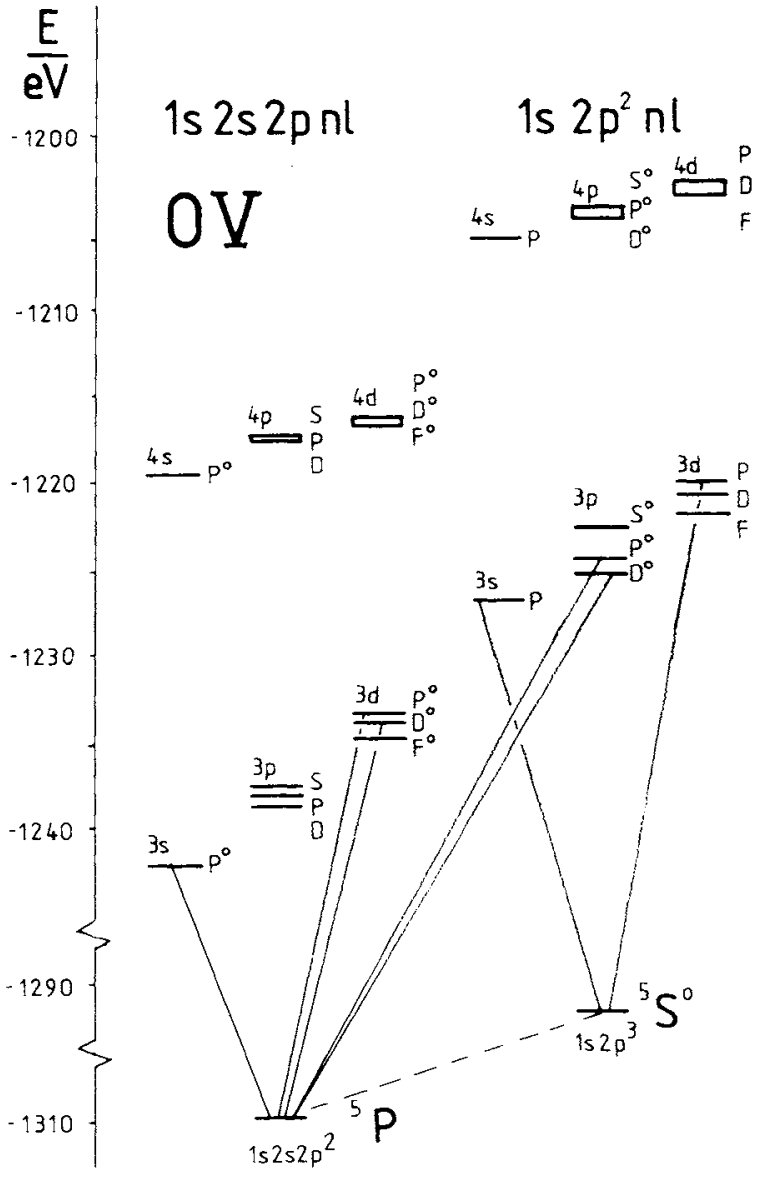

Fig. 1. Term diagram of doubly excited quintet states for the case of $\mathrm{O} V$. Full lines belong to transitions identified in the course of this work. The $1 s 2 s 2 p^{2}{ }^{5} P-1 s 2 p^{3}{ }^{5} S^{\circ}$ transition has been observed earlier.

$\mathrm{O}$, but it is very important for line identifications in $\mathrm{N}$ and has to be taken care of in a theoretical approach. This will be demonstrated when we discuss the line identifications in detail in section 5 .

The spectral resolution obtained in the experiment is high, but it is still insufficient to analyse fine structure. Therefore the position of the $L S$ terms is determined by averaging statistically over the calculated fine structure states.

The total energy obtained from these self-consistent calculations also includes the contributions of the Breit interaction, i.e. the magnetic interaction and retardation in perturbation theory. QED effects are taken into account by using the effective nuclear charge $Z_{\text {eff }}$ in the formulas of QED, which is calculated from an analogue hydrogenic orbital with the same expectation value of $r$ as the MCDF orbital in question.

The existing MCDF code was extended in order to be able to calculate transition probabilities, too. Upper and lower states of the transition were calculated with independent sets of one-electron orbitals. The consequent non-orthogonality of the orbitals was corrected partly by multiplying the transition matrix elements by the overlaps of the non-active orbitals. Differences of the lifetimes of different sublevels $J$ of a given $L S$-term typically turned out in a range of less than five percent. Again a statistical average over fine structure levels is carried out. Of the $n^{\prime}=3$ quintet levels, only the $n=2-n^{\prime}=3$ radiative decay channel was considered and therefore only partial lifetimes have been evaluated. However, according to the calculations by Chen [19], autoionization and intercombination decay to singly excited states can be neglected for the elements considered here.
This theoretical approach was tested for the lifetimes of doubly excited $n=3$ quartet states for elements $C$ to $F$. The results are in good agreement with model-potential calculations of Laughlin [20-22] and with experimental values $[23,24]$ gained by the beam-foil method [25]. We emphasize that mixing inside the complex is essential for most of the quartet transitions, but mixing with near perturbers with $n=4$ could safely be neglected for the $n=3$ states. This will be shown to be important for some quintet states with configuration in the discussion below.

\section{Experiment}

The experimental work was performed using the $4 \mathrm{MV}$ Dynamitron Tandem accelerator at the Ruhr-Universität Bochum. Stable ion beam currents of several $\mu \mathrm{A}$ could be obtained for the elements $\mathrm{N}, \mathrm{O}$ and $\mathrm{F}$. The ions were directed through thin carbon foils (of areal density of about $20 \mu \mathrm{g} \mathrm{cm}^{-2}$ ), produced by a glow discharge. Spectra were taken using a $2.2 \mathrm{~m}$ grazing-incidence monochromator (McPherson Mod. 247) which viewed the ion beam at right angles. A ruled diffraction grating with $600 \mathrm{l} / \mathrm{mm}$ and a holographic one with $36001 / \mathrm{m}$ were used alternatively in this experiment.

The linearity of the exit slit displacement of the monochromator was monitored by a $60 \mathrm{~mm}$ travel Heidenhain moiré fringe length gauge. A detailed description of the apparatus used can be taken from Träbert et al. [26].

Intensity decay curves were recorded for those quintet transitions which were not blended by other lines. In order to collect more signal, the more efficient grating with $600 \mathrm{l} / \mathrm{mm}$ groove density but lower spectral resolution was used. The carbon foil was moved in steps along the ion beam axis, monitored by another $60 \mathrm{~mm}$ travel Heidenhain moire fringe length gauge. The signal at each position was normalized to a preset value of integrated beam current.

A measured decay curve is a convolution of the exponential decay (due to the lifetime of the upper state) and the "detection window" (which is due to the finite spatial resolution of the detection system). The upper level is usually repopulated by cascading transitions. In a deconvolution procedure, three of four exponential functions and an assumed detection-window shape are fitted to the experimental decay curve. The main decay component is then identified with the lifetime of the upper level. For most of the levels studied here, cascading amounts to less than 5 percent of the amplitude of the main component. For all levels with strong cascading $\left(1 s 2 s 2 p 3 d^{5} P^{\circ},{ }^{5} D^{\circ}\right.$ and $1 s 2 p^{2} 3 d{ }^{5} P$ ), the lifetimes of the important cascades are known to be longer than the one of interest by at least one order of magnitude. Therefore we consider our fitting procedure justified even for these levels.

Starting values for the detection window parameters in the fit procedure can be obtained from geometrical considerations [27]. For very short lifetimes (under $15 \mathrm{ps),} \mathrm{the} \mathrm{width}$ of the wings of the detection window is of the same order of magnitude as the decay length of the main component. In these cases, it was not possible to fit both parameters independently. To overcome this problem, the form of the detection window was determined experimentally from a strong spectral line in a nearby wavelength region for which the lifetime is known and the cascade problem is similar to the case of the line of interest. By change this requirement is, for example, 


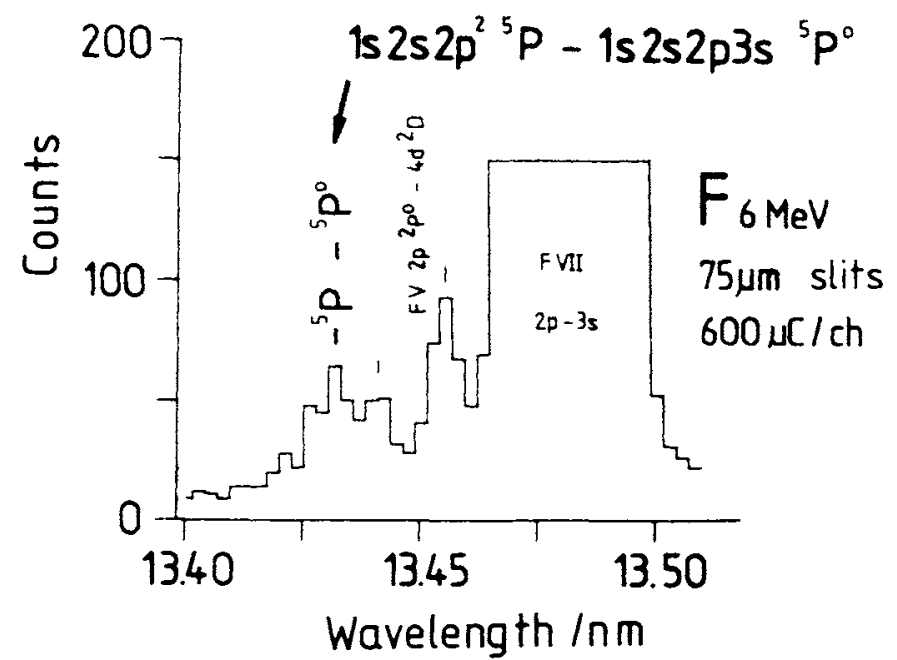

Fig. 2. Beam-foil spectrum of fluorine. Using a grating with $3600 \mathrm{l} / \mathrm{mm}$, the quintet transition F VI $1 s 2 s 2 p^{2}{ }^{5} P-1 s 2 s 2 p 3 s^{5} P^{\circ}$ can be resolved.

fulfilled for the transitions $1 s 2 s 2 p^{2}{ }^{5} P-1 s 2 s 2 p 3 d{ }^{5} P^{\circ},{ }^{5} D^{\circ}$ by the transition $1 s^{2} 2 p^{2} P^{o}-1 s^{2} 3 d^{2} D[14,28]$. Thus the window parameters can be kept fixed in the appropriate fitting procedure. Lifetimes even of the short-lived quintet states can then be determined, albeit with fairly large error bars.

\section{Transition $1 s 2 s 2 p^{2}{ }^{5} P-1 s 2 p 3 s{ }^{5} P^{0}$}

After investigating the transitions $1 s 2 s 2 p^{2}{ }^{5} P-1 s 2 s 2 p 3 d{ }^{5} P^{\circ}$, ${ }^{5} D^{\circ}[14]$ we studied the transition $1 s 2 s 2 p^{2}{ }^{5} P-1 s 2 s 2 p 3 s^{5} P^{\circ}$. As discussed in the theoretical part, we do not expect any near perturbers to have a notable influence on the states $1 s 2 s 2 p 3 s{ }^{5} P^{\circ}$ and $1 s 2 s 2 p 3 d{ }^{5} P^{\circ},{ }^{5} D^{\circ}$ (Fig. 1). However, for all three elements $\mathrm{N}, \mathrm{O}$ and $\mathrm{F}$, high resolution spectra were necessary to identify the transitions correctly. Figure 2 shows this exemplarily for the transition $1 s 2 s 2 p^{2}{ }^{5} P-1 s 2 s 2 p 3 s{ }^{5} P^{\circ}$ in fluorine. An attempt using the grating with $600 \mathrm{l} / \mathrm{mm}$ failed because of insufficient resolution. A spectrum recorded using the $36001 / \mathrm{mm}$ holographic grating, however, shows, besides the transition $\mathrm{F} \mathrm{V} 1 s^{2} 2 s^{2} 2 p^{2} P^{\circ}-1 s^{2} 2 s^{2} 4 d^{2} D$ at its expected position [28], another line at $(13.429 \pm 0.003) \mathrm{nm}$ that was identified with the above quintet transition. One should mention that both fine structure components of the $\mathrm{F} \mathrm{V}$ transition, $(1 / 2-3 / 2)$ and $(3 / 2-5 / 2 ; 3 / 2-3 / 2)$ show up with the relative intensity of $1: 2$ as expected in $L S$-coupling (Fig. 2).

For oxygen, even a measurement with the high-resolving power grating was not sufficient to resolve the quintet line from the transition $O$ IV $1 s^{2} 2 s 2 p^{2}{ }^{4} P-1 s^{2} 2 p^{2} 3 p^{4} S^{\circ}$ [28]. Measurements at different ion energies, comparing the line intensity with that of the transitions O IV $1 s^{2} 2 s 2 p^{2}{ }^{4} P$ $1 s^{2} 2 p^{2} 3 p^{4} P^{\circ}$, nevertheless confirmed the line at (18.344 \pm $0.005) \mathrm{nm}$ to originate from a transition in a four-electron ion.

In the case of nitrogen, spectra recorded with narrow slits ( $30 \mu \mathrm{m}$ wide) and using the $600 \mathrm{l} / \mathrm{mm}$ grating - the grating with $3600 \mathrm{l} / \mathrm{mm}$ can, for mechanical reasons, not be used in this wavelength region - allowed a proper assignment of the transition $1 s 2 s 2 p^{2}{ }^{5} P-1 s 2 s 2 p 3 s^{5} P^{\circ}$. This line can be sufficiently well resolved from the $1 s^{2} 2 p^{2} p^{\circ}-1 s^{2} 3 s^{2} S$ transition.

Table I shows the differences between these experimental transition energies and the theoretical values obtained from SC-calculations and those which incorporate all members of a complex. For the SC-approach the deviation is linear, for
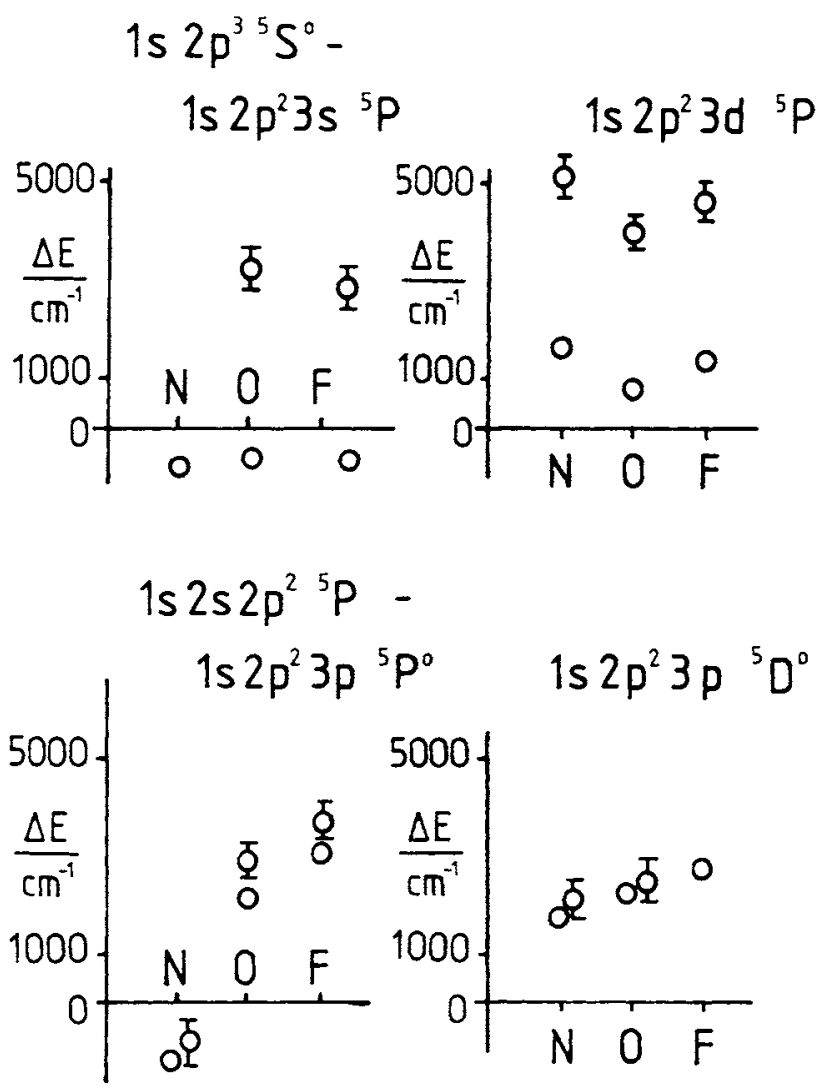

Fig. 3. Symbols with error bars: Deviation between experimental transition energies and those ones from a single-configuration approach. Symbols without error bars: Deviation between this SC-approach and an MCDFapproach which includes mixing in the complex and mixing between $1 s 2 p^{2} 3 l$ and $1 s 2 s 2 p 4 l$.

the complex approach even constant. Thus the assignments are confirmed.

\section{5. $1 s 2 p^{2} 3 l{ }^{5} L$ terms}

For $1 s 2 p^{2} 3 l^{5} L$ states one has to take into account the mixing with $1 s 2 s 2 p 4 l{ }^{5} L$ states. This is extremely important for the case of nitrogen. This mixing will destroy the linearity of the correlational part of the transition energies along an isoelectronic sequence. This concept was previously used for the identification of many quartet lines $[14,17]$, the quintet transitions $1 s 2 s 2 p^{2}{ }^{5} P-1 s 2 s 2 p 3 d{ }^{5} P^{\circ},{ }^{5} D^{\circ}[14]$ and for the line $1 s 2 s 2 p^{2}{ }^{5} P-1 s 2 s 2 p 3 s^{5} P^{\circ}$ as discussed in Section 4.

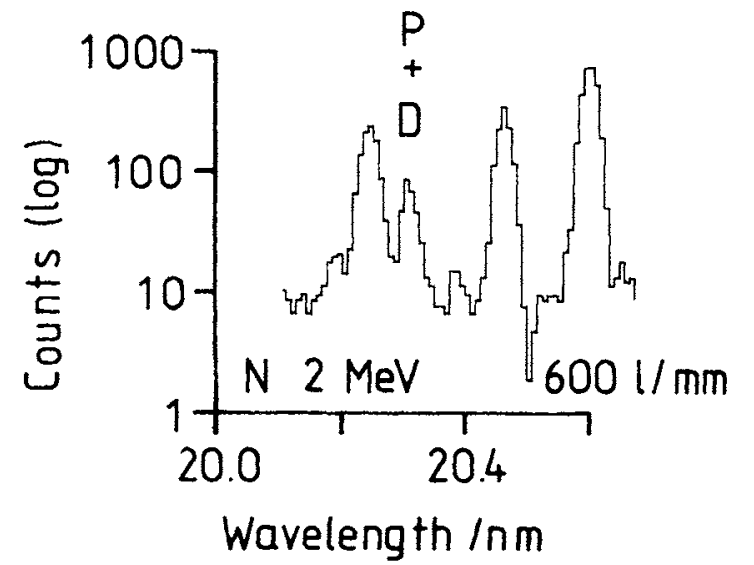

Fig. 4. Beam-foil spectrum of nitrogen recorded at an ion energy of $2 \mathrm{MeV}$. The line at $20.308 \mathrm{~nm}$ is identified as a blend of two quintet transitions: is $2 s 2 p^{2}{ }^{5} P-1 s 2 p^{2} 3 p{ }^{5} P^{\circ},{ }^{5} D^{\circ}$ 


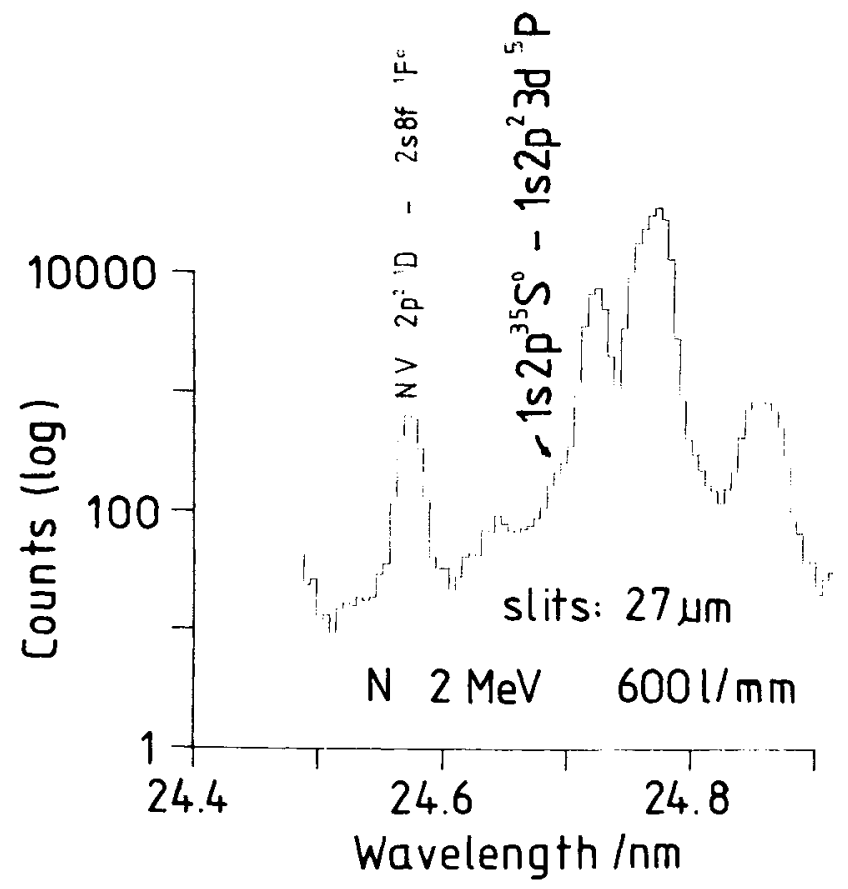

Fig. 5. Spectrum of nitrogen obtained using a $6001 / \mathrm{mm}$ grating. The resolution is good enough to identify the quintet line $1 s 2 p^{3}{ }^{5} S^{0}-1 s 2 p^{2} 3 d^{5} P$ at $24.691 \mathrm{~nm}$. At $24.573 \mathrm{~nm}$ Laughlin identified the $1 s^{2} 2 p^{2}{ }^{1} D-1 . s^{2} 2 s 8 f^{\prime} ' F^{\circ}$ transition [29].

In the following we consider transitions with a $1 s 2 p^{2}$ core of the upper state. These are: $1 s 2 s 2 p^{2}{ }^{5} P-1 s 2 p^{2} 3 p^{5} S^{\mathrm{O}}$, $1 s 2 s 2 p^{2}{ }^{5} P-1 s 2 p^{2} 3 p^{5} P^{\mathrm{o}}, 1 s 2 s 2 p^{2}{ }^{5} P-1 s 2 p^{2} 3 p^{5} D^{\circ}, 1 s 2 p^{3}{ }^{5} S^{\mathrm{o}}-$ $1 s 2 p^{2} 3 s^{5} P, 1 s 2 p^{35} S^{\mathrm{o}}-1 s 2 p^{2} 3 d^{5} P$.
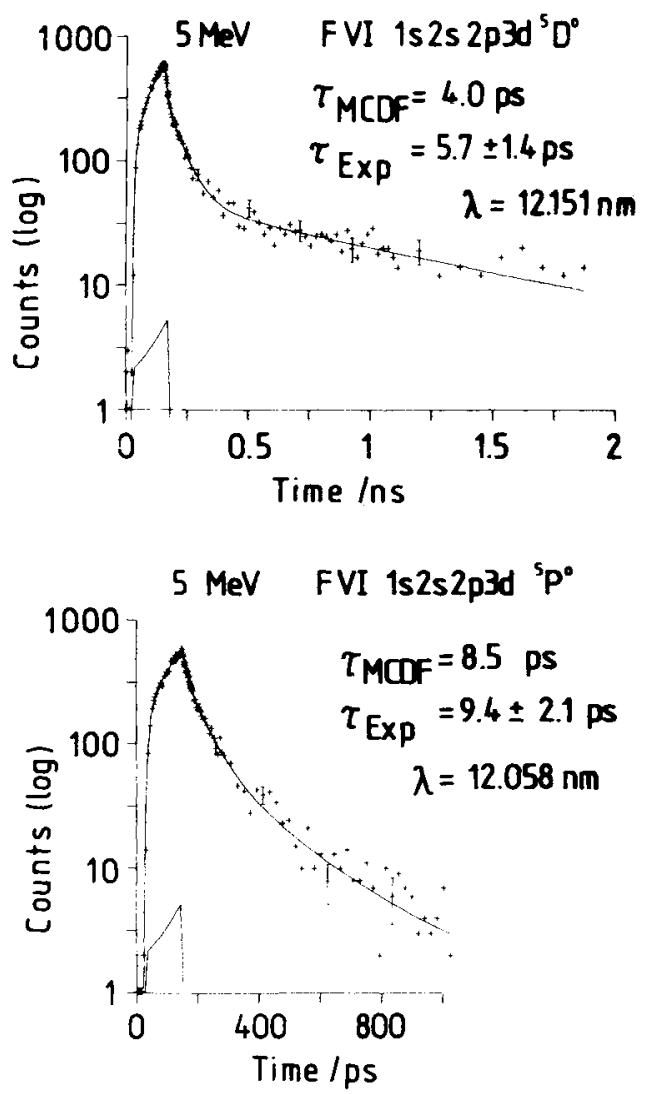

Fig. 6. Decay curves of two quintet transition: F VI $1 s 2 s 2 p^{2}{ }^{5} P-1 s 2 s 2 p 3 d^{5} P^{0}$, ${ }^{5} D^{\circ}$, recorded at a beam energy of $5 \mathrm{MeV}$ with spectrometer slits of $50 \mu \mathrm{m}$ The solid line is a fit curve with three exponentials convoluted with the detection system window function (also displayed). As expected, the curve with ${ }^{5} D^{0}$ as the upper state shows stronger cascading.

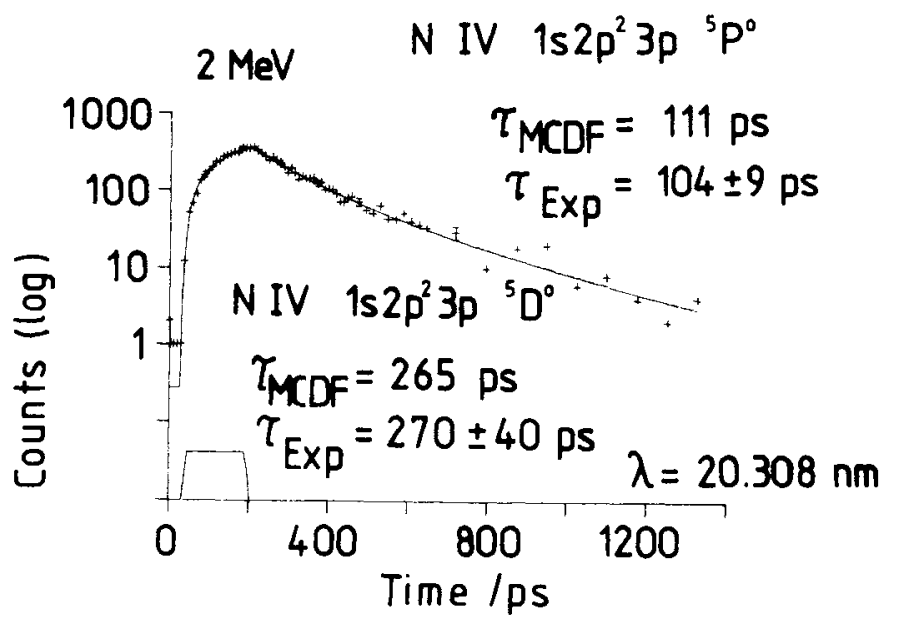

Fig. 7. Decay curves of the transition line at $20.308 \mathrm{~nm}$ (Fig. 4) taken at a beam energy of nitrogen ions of $2 \mathrm{MeV}$ and with slits of $60 \mu \mathrm{m}$ width. The analysis shows the presence of two strong compounds which, in agreement with our MCDF calculations, are identified with both transitions F VI $1 s 2 s 2 p^{2}{ }^{5} P-1 s 2 p^{2} 3 p^{s} P^{\circ},{ }^{5} D^{\circ}$.

The concept of our approach is to calculate the correlation energies of the upper states explicitly. This means that the mixing of all $n=3$-states in the complex is taken into account and that the mixing with the $1 s 2 s 2 p 4 l{ }^{5} L$ states is incorporated, too. This was done by first calculating the $n=3$ and $n=4$ states in their own complexes. Only those orbitals taking part in the pair correlation in question were not frozen in the subsequent common computer run. The inner orbitals were frozen from the $n=3$ states. The stability of convergence can be monitored [16]. This amount of extra correlation energy (circles without error bars) was then compared to the differences between experimental and theoretical transition energies of four transitions in question (Fig. 3). This latter difference (circles with error bars) is not linear for the two transitions as can be seen from the examples where three data points are available. In all cases the difference between the data marked by symbols, with and without error bars, is constant. This constant difference correlates with the lower $n=2$ state, and it is an indication that most of the missing, constant part of the correlation energy, which has not been calculated in the present approach, is due to the correlation energy of the lower state.

Some of the identifications have to be discussed in more detail. Both transitions N IV $1 s 2 s 2 p^{2}{ }^{5} P-1 s 2 p^{2} 3 p^{5} P^{\circ}$, $1 s 2 s 2 p^{2}{ }^{5} P-1 s 2 p^{2} 3 p^{5} D^{\circ}$ are identified with the line at $(20.308 \pm$ $0.007) \mathrm{nm}$ (Fig. 4), blending one another. The level N IV $1 s 2 p^{2} 3 p^{5} P^{\mathrm{o}}$ is shifted down from its unperturbed position by mixing with $1 s 2 s 2 p 4 s^{5} P^{\circ}$. Lifetime measurements confirm this and are discussed below.

The identification of the $1 s 2 s 2 p^{2}{ }^{5} P-1 s 2 p^{2} 3 p^{5} S^{0}$ transition for all three elements is only tentative. Although states of the form $1 s 2 s 2 p 4 l{ }^{5} S^{\circ}$ do not exist, and therefore no mixing with near perturbers is expected, the strong radial correlation with $1 s 2 p^{35} S^{0}$ cannot be handled in our approach. Furthermore, the line intensities are low as expected.

The transitions N IV $1 s 2 p^{3}{ }^{5} S-1 s 2 p^{2} 3 d^{5} P^{o}$ were found at $(24.691 \pm 0.006) \mathrm{nm}$ in the short-wavelength slope of the transition N IV $1 s^{2} 2 s^{2}{ }^{1} S-1 s^{2} 2 s 3 p^{1} P^{0}$ at $24.7205 \mathrm{~nm}$ [28] (Fig. 5). In this wavelength region the grating with $3600 \mathrm{l} / \mathrm{mm}$ cannot be used. In order to obtain sufficient spectral resolution with the $600 \mathrm{l} / \mathrm{mm}$ grating narrow slits of only $25 \mathrm{~mm}$ 
Table I. Comparison between experimental and theoretical results for the wavelengths of the quintet transition $1 s 2 s 2 p^{2}{ }^{5} P-$ $1 s 2 s 2 p 3 s{ }^{5} P^{\circ}$

\begin{tabular}{|c|c|c|c|c|c|}
\hline \multirow[b]{2}{*}{$Z$} & \multirow[b]{2}{*}{$\begin{array}{l}\text { Experiment } \\
\lambda / \mathrm{nm}\end{array}$} & \multicolumn{2}{|l|}{ Theory } & \multicolumn{2}{|l|}{ Deviation $\Delta E$} \\
\hline & & $\begin{array}{l}\text { "SC" } \\
\lambda / \mathrm{nm}\end{array}$ & $\begin{array}{l}\mathrm{MC} \text { in complex } \\
\lambda / \mathrm{nm}\end{array}$ & $\begin{array}{l}\text { "SC" } \\
\Delta E / \mathrm{cm}^{-1}\end{array}$ & $\begin{array}{l}\mathrm{MC} \text { in complex } \\
\Delta E / \mathrm{cm}^{-1}\end{array}$ \\
\hline 7 & $26.588 \pm 0.003$ & 26.570 & 26.804 & $-254 \pm 50$ & $3031 \pm 50$ \\
\hline 8 & $18.344 \pm 0.005$ & 18.321 & 18.450 & $-684 \pm 150$ & $3131 \pm 150$ \\
\hline 9 & $13.429 \pm 0.005$ & 13.408 & 13.487 & $-1166 \pm 170$ & $3202 \pm 170$ \\
\hline
\end{tabular}

"SC" means all configurations belonging to one single non-relativistic configuration.

"MC in complex" means that all configurations belonging to the complex are taken into account.

width were necessary. The line at $(24.573 \pm 0.003) \mathrm{nm}$ has been identified with N IV $1 s^{2} 2 p^{2}{ }^{1} D-1 s^{2} 2 s 8 f{ }^{\prime} F^{\circ}$ by Laughlin [29]. His calculation predicts a wavelength of $24.565 \mathrm{~nm}$. The appreciable line intensity is due to the strong mixing of $\mathrm{I} s^{2} 2 s 8 f^{\prime} F^{\circ}$ and $1 s^{2} 2 p 4 d^{i} F^{o}$. A lifetime measurement yielded

Table II. Summary of experimental and theoretical results for $n=2-n^{\prime}=3$ quintet transitions for the ions $N, O$ and $F$

\begin{tabular}{|c|c|c|}
\hline \multirow[b]{2}{*}{ Nitrogen $(Z=7)$} & \multicolumn{2}{|c|}{ Wavelength/nm } \\
\hline & $\mathrm{MCDF}(\mathrm{SC})$ & Experiment \\
\hline \multicolumn{3}{|l|}{$1 s 2 s 2 p^{2} s P$} \\
\hline$-1 s 2 s 2 p 3 s^{5} p^{0}$ & 26.570 & $26.588 \pm 0.003$ \\
\hline$-1 s 2 s 2 p 3 d^{5} p^{\circ}$ & 23.140 & $23.075 \pm 0.002^{\dagger}$ \\
\hline$-1 s 2 s 2 p 3 d^{5} D^{\circ}$ & 23.290 & $23.341 \pm 0.002^{\dagger}$ \\
\hline$-1 s 2 p^{2} 3 p^{5} S^{\circ}$ & 19.804 & $19.776 \pm 0.007$ \\
\hline$-1 s 2 p^{2} 3 p^{5} P^{\circ}$ & 20.273 & $20.308 \pm 0.007^{*}$ \\
\hline$-1 s^{2} p^{2} 3 p^{5} D^{0}$ & 20.404 & $20.308 \pm 0.007$ \\
\hline \multicolumn{3}{|l|}{$1 s 2 p^{3} S^{0}$} \\
\hline$-1 s 2 p^{2} 3 s^{5} P$ & 28.636 & \\
\hline$-1 s 2 p^{2} 3 d^{s} P$ & 24.994 & $24.961 \pm 0.005$ \\
\hline Oxygen $(Z=8)$ & $\operatorname{MCDF}(\mathrm{SC})$ & Experiment \\
\hline \multicolumn{3}{|l|}{$1 s 2 s 2 p^{2}{ }^{5} P^{\mathrm{o}}$} \\
\hline$-1 s 2 s 2 p 3 s^{5} P^{0}$ & 18.321 & $18.344 \pm 0.005$ \\
\hline$-1 s 2 s 2 p 3 d^{5} P^{o}$ & 16.254 & $16.227 \pm 0.002^{\dagger}$ \\
\hline$-1 s 2 s 2 p 3 d^{5} D^{0}$ & 16.338 & $16.380 \pm 0.002^{\dagger}$ \\
\hline$-1 s 2 p^{2} 3 p^{5} S^{\circ}$ & 14.281 & $14.271 \pm 0.007^{*}$ \\
\hline$-1 s 2 p^{2} 3 p^{5} p^{0}$ & 14.598 & $14.536 \pm 0.003$ \\
\hline$-1 s 2 p^{2} 3 p^{5} D^{11}$ & 14.683 & $14.628 \pm 0.003$ \\
\hline \multicolumn{3}{|l|}{$1 s 2 p^{3} S^{0}$} \\
\hline$-1 s 2 p^{2} 3 s^{5} P$ & 19.488 & $19.359 \pm 0.004$ \\
\hline$-1 s 2 p^{2} 3 d^{5} P$ & 17.319 & $17.200 \pm 0.003$ \\
\hline Fluorine $(Z=9$ ) & $\operatorname{MCDF}(\mathrm{SC})$ & Experiment \\
\hline \multicolumn{3}{|l|}{$1 s 2 s 2 p^{2}{ }^{5} P$} \\
\hline$-1 s 2 s 2 p 3 s^{5} P^{0}$ & 13.408 & $13.429 \pm 0.003$ \\
\hline$-1 s 2 s 2 p 3 d^{5} p^{0}$ & 12.066 & $12.058 \pm 0.002^{\dagger}$ \\
\hline$-1 s 2 s 2 p 3 d^{5} D^{\circ}$ & 12.118 & $12.151 \pm 0.002^{\dagger}$ \\
\hline$-1 s 2 p^{2} 3 p^{5} S^{1}$ & 10.795 & $10.798 \pm 0.005^{*}$ \\
\hline$-1 s 2 p^{2} 3 p^{5} p^{0}$ & 11.020 & $10.976 \pm 0.003$ \\
\hline$-1 s 2 p^{2} 3 p^{5} D^{0}$ & 11.078 & \\
\hline \multicolumn{3}{|l|}{$1 s 2 p^{35} S^{0}$} \\
\hline$-1 s 2 p^{2} 3 s^{5} P$ & 14.132 & $14.076 \pm 0.004$ \\
\hline$-1 s 2 p^{2} 3 d^{5} P$ & 12.734 & $12.659 \pm 0.003$ \\
\hline
\end{tabular}

* Tentative assignment.

+ Already published [14].

Physica Scripta 44
$(104 \pm 4)$ ps which is supported by Laughlin's calculated value of $124 \mathrm{ps}$.

An extrapolation according to Fig. 3 predicts the transition N IV $1 s 2 s 2 p^{2}{ }^{5} P-1 s 2 p^{2} 3 p{ }^{5} D^{\circ}$ to give rise to a line at $(11.11 \pm 0.01) \mathrm{nm}$. Even with the $3600 \mathrm{l} / \mathrm{mm}$ grating, it was not possible to resolve it from the quartet transition $1 s 2 p^{2}{ }^{4} P$ $1 s 2 p 3 d^{4} D^{\circ}$ at $(11.111 \pm 0.005) \mathrm{nm}$ [30]. Similarly, the transition N IV $1 s 2 p^{3}{ }^{5} S-1 s 2 p^{2} 3 s^{5} P^{\circ}$ expected at $(28.43 \pm 0.02 \mathrm{~nm})$ is apparently blended by the lines N IV $1 s^{2} 2 s 2 p^{3} P^{0}-$ $1 s^{2} 2 s 3 d^{3} D$ at $28.351 \mathrm{~nm}$ [28] and N III $1 s^{2} 2 s 2 p^{2}{ }^{4} P-$ $1 s^{2} 2 s 2 p 5 d^{4} D^{\circ}$ at $28.433 \mathrm{~nm}[28]$.

Table II lists all quintet identifications obtained by our approach for the elements $\mathrm{N}, \mathrm{O}$ and $\mathrm{F}$. In addition to

Table III. Partial lifetimes of doubly excited four-electron $n=3$ quintet states due to optical transitions to $n=2$ states

\begin{tabular}{|c|c|c|c|c|}
\hline \multirow[b]{2}{*}{ Element } & \multicolumn{2}{|l|}{ Experiment } & \multicolumn{2}{|c|}{ Theory (MCDF) } \\
\hline & $\begin{array}{l}\text { Quintet state } \\
\tau / \mathrm{ps}\end{array}$ & $\begin{array}{l}\text { "SC" } \\
\tau / \mathrm{ps}\end{array}$ & $\begin{array}{l}\text { "MC } \\
\text { in complex" } \\
\tau / \mathrm{ps}\end{array}$ & $\begin{array}{l}\text { Mixing with } \\
n=4,1 s 2 s 2 p 4 l \\
\tau / p s\end{array}$ \\
\hline \multicolumn{5}{|c|}{$1 s 2 s 2 p 3 d^{5} P^{0}$} \\
\hline $\mathrm{N}$ & $33.2 \pm 3.2$ & 33.4 & 33.4 & \\
\hline$O$ & & 15.1 & 14.9 & \\
\hline$F$ & $9.4 \pm 2.1$ & 7.9 & 7.7 & \\
\hline \multicolumn{5}{|c|}{$1 s 2 s 2 p 3 d{ }^{5} D^{\circ}$} \\
\hline $\mathrm{N}$ & $18.1 \pm 1.2$ & 19.3 & 17.1 & \\
\hline $\mathrm{O}$ & $6.3 \pm 2.3$ & 8.7 & 7.7 & \\
\hline $\mathrm{F}$ & $5.7 \pm 1.4$ & 4.5 & 4.0 & \\
\hline \multicolumn{5}{|c|}{$1 s 2 p^{2} 3 s^{5} P$} \\
\hline $\mathrm{N}$ & & 427 & 219 & \\
\hline $\mathrm{O}$ & & 213 & 102 & \\
\hline $\mathrm{F}$ & $65 \pm 7$ & 119 & 55 & \\
\hline \multicolumn{5}{|c|}{$1 s 2 p^{2} 3 d^{5} P$} \\
\hline $\mathrm{N}$ & & 21.5 & 22.1 & 22.7 \\
\hline $\mathrm{O}$ & & 9.3 & 9.6 & 9.5 \\
\hline $\mathrm{F}$ & $7.2 \pm 1.5$ & 4.7 & 4.8 & 4.9 \\
\hline \multicolumn{5}{|c|}{$1 s 2 p^{2} 3 p^{5} P^{\circ}$} \\
\hline $\mathrm{N}$ & $104 \pm 9$ & 73 & 139 & 111 \\
\hline $\mathrm{O}$ & & 34 & 66 & 58 \\
\hline $\mathrm{F}$ & $35 \pm 4$ & 18 & 35 & 30.5 \\
\hline \multicolumn{5}{|c|}{$1 s 2 p^{2} 3 p^{5} D^{\circ}$} \\
\hline $\mathrm{N}$ & $270 \pm 40$ & 73 & 248 & 261 \\
\hline $\mathrm{O}$ & & 34 & 143 & 153 \\
\hline $\mathrm{F}$ & & 18.3 & 79 & 84 \\
\hline
\end{tabular}

"SC" includes all configurations which belong to one single non-relativistic configuration.

"MC in complex" means that all configurations belonging to the complex are taken into account. 
the experimental wavelength values, results of "Singleconfiguration" MCDF calculations are included.

\section{Lifetimes of quintet states}

For all observed and identified quintet transitions, lifetime measurements as described in Section 3 were attempted. For a number of transitions this turned out to be unsuccessful because of severe blending or low intensity of the transitions in question. For example, in the case of oxygen we were only able to investigate the state $1 s 2 s 2 p 3 d{ }^{5} D^{\circ}$ without such problems.

As discussed above for the case of short lifetimes below about $15 \mathrm{ps}$, some of the window function parameters had to be fixed during the fit procedure. The detection window parameters can be experimentally determined from strong transitions where the lifetime is reliably known (e.g. $\left.1 s^{2} 2 p^{2} P^{\circ}-1 s^{2} 3 d^{2} D\right)$. This procedure, however, leads to results which carry larger error bars, especially for the states O V $1 s 2 s 2 p 3 d^{5} D^{\circ}$, F VI $1 s 2 s 2 p 3 d^{5} P^{\circ},{ }^{5} D^{\circ}$ and F VI $1 s 2 p^{2} 3 d{ }^{5} P$.

Another indication concerning the correct identification of the lines is provided by cascades which appear in the case of the transition studied. Decay curves taken for the state $1 s 2 s 2 p 3 d^{5} D^{\circ}$ definitely show up with stronger cascading than those for the level $1 s 2 s 2 p 3 d{ }^{5} P^{\circ}$. This is demonstrated in Fig. 6 which shows the decay curves of the transitions F VI 1s $2 s 2 p^{2}{ }^{5} P-1 s 2 s 2 p 3 d{ }^{5} P^{\circ},{ }^{5} D^{\circ}$, respectively. Strong cascades were also found when measuring the lifetime of the F VI $1 s 2 p^{2} 3 d{ }^{5} P$ level.

As mentioned above, the nitrogen line at $23.308 \mathrm{~nm}$ is identified as a blend of two quintet transitions, N IV $1 s 2 s 2 p^{2}{ }^{5} P-1 s 2 p^{2} 3 p^{5} P^{0}$ and $1 s 2 s 2 p^{2}{ }^{5} P-1 s 2 p^{2} 3 p^{5} D^{\circ}$. This hypothesis is confirmed by the analysis of the decay curve taken at this wavelength (Fig. 7)). There are two strong decay components present in the data, with decay times which are in agreement with the MCDF-results. In the case of the $1 s 2 p^{2} 3 p^{5} P^{0}$ level the theoretical value takes into account not only mixing in the complex but also mixing with $1 s 2 s 2 p 4 s$.

The nitrogen line at $26.543 \mathrm{~nm}$ also contains two components. The main component supports the identification of the quintet transition N IV $1 s 2 p^{3} S-1 s 2 p^{2} 3 s^{5} P^{\circ}$. The second (short-lived) component of $(12 \pm 5) \mathrm{ps}$ is assigned tentatively to the sextet transition $1 s 2 s 2 p^{3}{ }^{6} S^{\circ}-1 s 2 s 2 p^{2} 3 d^{6} P$. MCDF calculations indicate a lifetime of $6.7 \mathrm{ps}$. This sextet transition will be discussed in detail in a forthcoming paper [31].

A summary of experimental and theoretical lifetimes is given in Table III. The theoretical results are only partial lifetimes which relate to the radiative decay branch to $n=2$ quintet states only. Autoionization and intercombination decay (by change of spin) to singly excited states are neglected.

\section{Conclusion}

We were able to identify a number of $n=2-n^{\prime}=3$ transitions in the quintet term systems of N IV, O V and F VI. This was achieved by comparing the deviations between the experimental and calculated transition energies and analysing smooth trends of these deviations along the isoelectronic sequence. Term energy shifts due to near perturbers were handled qualitatively in the calculations and are confirmed by the experimental observations. The identifications of a number of quintet transitions were corroborated by the analysis of decay curves. The lifetimes obtained largely agree with results from MCDF calculations.

\section{Acknowledgements}

We thank the staff of the Bochum accelerator for the stable and intense ion beams. The excellent exciter foils were prepared by H. H. Bukow, whilst C. Wagner helped with the experiment. The data acquisition system used was granted by the Deutsche Forschungsgemeinschaft. We would like to thank C. Laughlin for the many helpful discussions and especially for identifying the nitrogen line at $24.573 \mathrm{~nm}$. Discussions with and help by T. Brage (Lund, Nashville) are gratefully acknowledged. Finally we would like to express our thanks to J. P. Desclaux for his continuous support. One of us (J. H. B) is grateful to the Deutsche Forschungsgemeinschaft (DFG) and the Gesselschaft für Schwerionenforschung Darmstadt (GSI) for financial support. D. H. is grateful to the Deutsche Forschungsgemeinschaft (DFG) for a post-doc scholarship.

\section{References}

1. Mannervik, S., Physica Scripta 40, 28 (1989).

2. Mannervik, S., Astner, G. and Kieselinski, M., J. Phys. B13, L441 (1980); Brooks, R. L., Hardis, J. E., Berry, H. G., Curtis, L. J., Cheng, K. T. and Ray, W., Phys. Rev. Lett. 45, 1318 (1980).

3. Andersen, T. unpublished work (1980) quoted in Brooks, R. L., Hardis, J. E., Berry, H. G., Curtis, L. J., Cheng, K. T. and Ray, W., Phys. Rev. Lett. 45, 1318 (1980).

4. Beck, D. R. and Nicolaides, C. A. Phys. Lett. 61A, 227 (1977); Martinson, I., Bickel, W. S. and Ölme, A., J. Opt. Soc. Am. 60, 1213 $(1970)$.

5. Brooks, R. L., Hardis, J. E., Berry, H. G., Curtis, L. J., Cheng, K. T. and Ray, W., Phys. Rev. Lett. 45, 1318 (1980).

6. Martinson, 1., Denne, B., Ekberg, J. O., Engström, L., Huldt, S., Jupen, C., Litzen, U., Mannervik, S. and Trigueiros, A., Physica Scripta 27, 201 (1983).

7. Hardis, J. E., Berry, H. G., Curtis, L. J. and Livingston, A. E., Physica Scripta 30, 189 (1984).

8. Träbert, E., Jahresbericht Dynamitron-Tandem-Laboratorium (Bochum) 13, 48 (1986).

9. Bunge C. F., Phys. Rev. Lett. 44, 1450 (1982).

10. Brage, T. and Froese Fischer, C., J. Phys. B21, 2563 (1988).

11. Chung, K. T., Phys. Rev. A40, 4203 (1989).

12. Agentoft, M., Andersen, T., Froese Fischer, C. and Smentek Mielszarek, L., Physica Scripta 28, 45 (1983).

13. Hontzeas, S., Martinson, I., Erman, P. and Buchta, R., Physica Scripta 6, 55 (1972).

14. Blanke, J. H., Fricke, B., Sepp, W. -D., Heckmann, P. H., Möller, G. and Wagner, C., Physica Scripta 42, 522 (1990).

15. Desclaux, J. P., Comp. Phys. Comm. 9, 31 (1975).

16. Desclaux, J. P., MCDF version 1989 (private communication).

17. Blanke, J. H., Fricke, B., Heinemann, D., Sepp, W. D., Thies, B., Göbenli, T., Heckmann, P. H., Möller, G. and Träbert, E., Z. Phys. D13, 1 (1989).

18. Brage, T., PhD Thesis, Lund (1988).

19. Chen, M. H., Atomic Data and Nuclear Data Tables 34, 301 (1986).

20. Laughlin, C., Z. Phys. D9, 273 (1988).

21. Laughlin, C., Z. Phys. D16, 9 (1990).

22. Laughlin, C. and Fairley, N. A., Z. Phys. D5, 91 (1987).

23. Schoch, R., Diplomarbeit, Freiburg (1988).

24. Blanke, J. H., Heckmann, P. H. and Trävert, E., Physica Scripta 32, 509 (1985).

25. Blanke, J. H., Dissertation, Kassel (1990).

26. Träbert, E., Heckmann, P. H., Schlagheck, W. and Buttlar, H. V., Physica Scripta 21, 27 (1980).

27. Träbert, E., Dissertation, Bochum (1978).

28. Kelly, R. L., Atomic and Ionic Spectrum Lines Below 2000 A. Hydrogen through Krypton, Oak Ridge National Laboratory Report ORNL5922 (1982).

29. Laughlin, C., Private communication (1990).

30. Blanke, J. H., Heckman, P. H., Träbert, E. and Hucke, R., Physica Scripta 35, 780 (1987).

31. Brage, T., Miecznik, G., Blanke, J. H., Fricke, B. and Heckmann, P. H. (In preparation) 